\title{
The impact of SARS-CoV-2 (COVID-19) pandemic on trauma bay management and guideline adherence in a European level-one-trauma centre
}

\author{
Sascha Halvachizadeh ${ }^{1,2}$ (D) Michel Teuben ${ }^{1,2} \cdot$ Till Berk $^{1} \cdot$ Valentin Neuhaus $^{1} \cdot$ Hans-Christoph Pape $^{1,2}$. \\ Roman Pfeifer ${ }^{1,2}$
}

Received: 18 June 2020 / Accepted: 16 July 2020 / Published online: 28 July 2020

(C) The Author(s) 2020

\begin{abstract}
Purpose SARS CoV-2 (COVID-19) represents a pandemic that has led to adjustments of routine clinical practices. The initial management in the trauma bay follows detailed international valid algorithms. This study aims to work out potential adjustments of trauma bay algorithms during a global pandemic in order to reduce contamination and to increase safety for patients and medical personnel.

Methods This retrospective cohort study compared patients admitted to the trauma bay of one academic level-one trauma centre in March and April 2019 with patients admitted in March and April 2020. Based on these datasets, possible adjustments of the current international guidelines of trauma bay management were discussed.

Results Group Pan $(2020, n=30)$ included two-thirds the number of patients compared with Group Ref (2019, $n=44)$. The number of severely injured patients comparable amongst these groups: mean injury severity score (ISS) was significantly lower in Group Pan (10.5 \pm 4.4 points) compared with Group Ref (15.3 \pm 9.2 points, $p=0.035)$. Duration from admission to whole-body CT was significantly higher in Group Pan (23.8 \pm 9.4 min) compared with Group Ref $(17.3 \pm 10.7 \mathrm{~min}, p=0.046)$. Number of trauma bay admissions decreased, as did the injury severity for patients admitted in March and April 2020. In order to contain spreading of SARS Cov-2, the suggested recommendations of adjusting trauma bay protocols for severely injured patients include (1) minimizing trauma bay team members with direct contact to the patient; (2) reducing repeated examination as much as possible, with rationalized use of protective equipment; and (3) preventing potential secondary inflammatory insults.

Conclusion Appropriate adjustments of trauma bay protocols during pandemics should improve safety for both patients and medical personnel while guaranteeing the optimal treatment quality. The above-mentioned proposals have the potential to improve safety during trauma bay management in a time of a global pandemic.
\end{abstract}

Keywords Trauma bay management · Severe trauma $\cdot$ COVID-19 · SARS CoV-2 · Polytrauma management

Level of evidence: Level III, Retrospective comparative study without negative criteria, Therapeutic/Care Management

Sascha Halvachizadeh

Sascha.Halvachizadeh@usz.ch

Michel Teuben

Michel.Teuben@usz.ch

Till Berk

Till.Berk@usz.ch

Valentin Neuhaus

Valentin.Neuhaus@usz.ch
Hans-Christoph Pape

Hans-Christoph.Pape@usz.ch

Roman Pfeifer

Roman.Pfeifer@usz.ch

1 Department of Trauma, University Hospital Zurich, Rämistrasse 100, 8091 Zurich, Switzerland

2 Harald-Tscherne laboratory for orthopedic and trauma research, University of Zurich, Sternwartstrasse 14, 8091 Zurich, Switzerland 


\section{Background}

Trauma is amongst the top ten causes of death worldwide [1]. Numerous international guidelines have been introduced worldwide in order to standardize the treatment of severely injured patients and to improve their survival [2-4]. These guidelines include recommendations for the minimum number of required medical professionals in the trauma bay (e.g., anesthesiologists, traumatologist, neurosurgeons, nurses, technicians etc..), initial assessments according to Advanced Trauma Life Support (e.g., blood pressure measurements, respiratory rate, auscultation and examination of body regions, etc.), and settings around the trauma bay management, such as operation room or computer tomography availability $[5,6]$. SARS CoV-2, a rapidly spreading pandemic, has led to increasing infection and mortality rates which threatens to overwhelm the critical care infrastructure [7]. Several measures have recently been implemented in order to optimize treatment and outcomes of COVID-19 patients. Both intramural (mandated quarantine of medical personnel, novel isolation wards, involvement of other physicians from other specialties in daily COVID-19 care) and extramural (businesses have been closed, social distancing, isolation) measures have been introduced. Reports documenting infections have shown symptoms or even mortalities of medical personnel (nurses and doctors) and healthcare workers exacerbating the crisis [8]. Additional stresses include the increased need for medical protective equipment and medical supplies. Without a doubt, the increased spread of the COVID-19 over the population and lower capacities in critical care infrastructure directly affects the management and strategies in treatment of severely injured patients.

This study aims to analyze the impact of the global SARS CoV-2 pandemic on the treatment of patients in the trauma bay in a level-one trauma center. Based on the challenges brought by SARS CoV-2, this study further discusses potential adjustments of trauma bay guidelines:

1. How to comply with international recommendations and guidelines and reduce risks of infections in medical personal (surgeons, nurses, emergency practitioners) involved in trauma management.

2. How to maintain the quality of trauma management without being contaminated or spreading the virus throughout the hospital.

3. How to protect of critical care infrastructure from becoming overwhelmed.

\section{Methods}

The local institutional review board and the cantonal ethics committee received a detailed study protocol and waved the requirement for ethical approval to conduct this study (Nr. 2020-00789). This retrospective cohort study was conducted under adherence to the STROBE guidelines [9].

\section{Study population and data collection}

Data from patients who were treated in the trauma bay of one academic level-one trauma center was extracted from the electronic patient charts. For the purpose of the study, two study groups were composed based on admission data:

- Group Pan(demic): included all patients admitted during the COVID-19 pandemic period between Mar 01, 2020 and Apr 15, 2020.

- Group Ref(erence) was composed to serve as a reference group and included all patients admitted between Mar 01, 2019 and Apr 15, 2019.

Exclusion criteria were death in the trauma bay, secondary admissions to the trauma from other hospitals, or international repatriations. This study compared patients treated in the same month to increase seasonal comparability and to reduce selection bias. The patients included were treated for the following reason: The Swiss Federal Department of Health recommended in March 2020 behavioral changes of the population to reduce the spreading of SARS CoV-2. These recommendations included social distancing of $2 \mathrm{~m}(6 \mathrm{ft})$, staying at home as much as possible, and short-time contact to other persons (15 min). In addition, non-essential businesses have been closed. For clinical personnel, wearing of personal protective equipment, such as gloves, masks, coats, and glasses has been introduced.

\section{Definitions}

The Injury Severity Score (ISS) was calculated based on information found on the discharge paper in common manner [10]. Severely injured patients were defined as ISS $\geq 16$ points [2]. Total duration in the trauma bay indicates the time from admission to the trauma bay to the time of discharge to either the operating room (OR), intensive care unit (ICU), or the ward. The time from admission to whole-body $\mathrm{CT}$ scan (WBCT) represents the delivery procedure, an initial assessment, and the preparation of the trauma team and the patient for WBCT. Life-saving interventions included emergency intubation, placement of a chest tube, laparotomy, craniotomy, thoracotomy, external fixation of pelvic fracture, and other interventions to stop major bleedings in the trauma bay. Injury mechanisms included fall from any height, motor vehicle accidents (MVAs), blunt trauma from other causes (violent attacks), penetrating injuries, or suicide attempts. Treatment and diagnostics as well as trauma team members followed the local and international guidelines for trauma resuscitation [6]. 


\section{Parameters studied}

Collected variables included patient demographics (age, gender), ISS, total duration spent in the trauma bay, duration from admission WBCT, number of severely injured patients, and life-saving interventions performed in the trauma bay.

\section{Statistical analysis}

Continuous variables were summarized as mean \pm standard deviation and discrete variables as a number and percentage. Group comparison was performed using Student's $t$ test on continuous and Pearson's chi-square test on discrete variable with $95 \%$ confidence interval (CI) in case of normal distribution. Skewed distributed variables were compared using Mann-Whitney $U$ test. A $p$ value of below 0.05 was considered statistically significant. All statistical analyses were performed using R (R Core Team (2018). R: A language and environment for statistical computing. R Foundation for Statistical Computing, Vienna, Austria. URL https://www.Rproject.org/).

\section{Results}

\section{Patient characteristics}

A total of 76 patients were initially identified. One patient was excluded in each study group, due to death before finishing trauma bay diagnostics. In Group Ref, one patient died after a self-inflicted gunshot to the head, and in Group Pan, one patient died due to brain herniation after suicidal hanging.

The final cohort included 74 patients, mean age 50.0 years ( \pm 21.8$)$, with a majority being male $(n=54,73.0 \%)$ (Table 1$)$. Group Ref included 44 (59.4\%) patients, while Group Pan included $30(40.5 \%)$ patients. Demographic variables including age and gender distribution were comparable amongst these groups.

Table 1 Included patients

\begin{tabular}{lc}
\hline Number & 74 \\
Age (years), mean (SD) & $50.0(21.8)$ \\
Male, $n(\%)$ & $54(73.0)$ \\
Patients during COVID-19 Pandemic, $n(\%)$ & $30(40.5)$ \\
ISS (points), mean (SD) & $12.2(8.3)$ \\
Severely injured patients, $n(\%)$ & $22(29.7)$ \\
Total duration in trauma bay (min), mean (SD) & $83.3(30.4)$ \\
Duration from admission to WBCT (min), mean (SD) & $19.1(10.3)$ \\
\hline
\end{tabular}

$S D$ standard deviation, ISS Injury Severity Score, WBCT whole-body CT
Injury severity and mechanism

Falls were the most common injury mechanism (Group Ref $n=23(52.3 \%)$ vs. Group Pan $n=17$ (56.7\%), 0.912), followed by motor vehicle accidents (Group Ref $n=16$ (36.4\%) vs. Group Pan $n=11$ (36.7\%), 0.912). The number of severely injured patients in both groups was comparable (Group Ref $n=15$ (34.1\%) versus Group Pan $=7$ (23.3\%), 0.462). However, the mean ISS was significantly higher in Group Ref (15.3 \pm 9.2 points) compared with Group Pan (10.5 \pm 64.4 points, $95 \%$ CI 0.8 to $6.4, p=0.035$ ) (Table 2).

\section{Trauma bay times}

The total duration in the trauma bay for Group Ref was $76.2 \pm$ 29.6 minutes while in Group Pan, the total duration was 93.6 $\pm 28.9 \mathrm{~min}$. This difference was statistically significant (95\% CI -31.2 to $-3.5,0.014)$.

The duration from admission to WBCT for Group Ref was $17.3 \pm 10.7$ minutes. This was found to be significantly lower when compared with Group Pan (23.8 \pm 9.4 minutes, $95 \%$ CI -9.2 to $-1.2, p=0.046$ ).

\section{Lifesaving interventions}

The total number of life-saving interventions per patient in the trauma bay was comparable amongst Group Ref and Group Pan $(0.6 \pm 0.9$ vs. $0.4 \pm 0.8,95 \% \mathrm{CI}-0.2$ to $0.6,0.31)$. After treatment in the trauma bay, the patients were frequently dismissed to the ICU (Group Ref $n=25$ (56.8\%) vs Group Pan $n=18$ (60\%), 0.974).

\section{Discussion}

The SARS CoV-2 pandemic challenges routine clinical care and more specifically largely interferes with trauma care. In order to optimize planning of trauma care in the near future, there is a need to define specific alterations and to identify practical pitfalls. To do so, the current study aimed to compare trauma bay characteristics under COVID-19 pandemic conditions and during a corresponding time period under regular circumstances (prior to COVID-19 pandemic). The following differences were identified:

- During the pandemic, there were $31.2 \%$ less patients treated in the trauma bay

- Despite a comparable number of severely injured patients, the Injury Severity Score was significantly lower during the pandemic

- Both the total duration in the trauma bay and the duration from admission to WBCT were significantly higher during the pandemic 
Table 2 Comparison of time spent in trauma bay

\begin{tabular}{llll}
\hline & Group Ref & Group Pan & $p$ value \\
\hline$n$ & 44 & 30 & \\
Age (years), mean (SD) & $50.7(23.1)$ & $48.9(20.1)$ & 0.739 \\
Male, $n$ (\%) & $32(72.7)$ & $22(73.3)$ & 1 \\
ISS (points), mean (SD) & $15.3(9.2)$ & $10.5(4.4)$ & 0.035 \\
Severely injured patients, $n(\%)$ & $15(34.1)$ & $7(23.3)$ & 0.462 \\
Total duration in trauma bay (min), mean (SD) & $76.2(29.6)$ & $93.6(28.9)$ & 0.014 \\
Duration from admission to WBCT (min), mean (SD) & $17.3(10.7)$ & $23.8(9.4)$ & 0.046 \\
\hline
\end{tabular}

$n$ number, $S D$ standard deviation, ISS Injury Severity Score, $W B C T$ whole-body CT
The observed decrease in trauma bay admissions during the COVID-19 pandemic is likely associated with the restrictions introduced by the federal health department. Individuals were recommended to avoid unnecessary movements and expected to stay at home, as much as possible. Furthermore, nonessential businesses have been closed. Consequently, frequencies of both labor-related injuries, violence, and traffic incidents were likely lower than during normal conditions. It is assumed that admission rates of trauma patients have been influenced by patients avoiding trauma submissions.

The data collected in this study revealed that "Group Pan" had significantly lower ISS, compared to the reference group from the year 2019 "Group Ref." It is tempting to hypothesize that increased awareness and alertness of the general population might have affected behaviors towards high-risk sports or driving habits. However, the number of severely injured patients was comparable between the groups, indicating the necessity of maintaining the same quality of care despite the decrease of ISS.

Even with a reduced injury severity, an increased duration from admission to CT scan was observed. These changes are difficult to explain; however, they might be a result of increased alertness of medical personnel, associated with increased hygiene recommendations and personal protection measures. The increased caution of self-exposure towards potentially infected people during daily living might also have affected medical personnel during their routine clinical treatment.

Based on these changes and the developments during the SARS CoV-2 crisis, the following points should be discussed regarding potential adjustments to existing trauma bay guidelines (Table 3):

\section{Medical personnel in trauma bay}

Recent guidelines (Level 3 Guidelines on the treatment on patients with severe/multiple injuries by European Society of Trauma and Emergency Surgery (ESTES)) indicate that the basic trauma team must consist of at least three physicians ( 2 surgeons and 1 anesthesiologist) [6,11]. Local conditions of the trauma system in each region usually define which specialties are primarily present in the emergency department. Other disciplines, such as neurosurgeons or/and radiologist, and nurses/technicians from each department are frequently present in emergency department during the initial assessment of the patient in the trauma bay. In the time of pandemics, such as COVID-19, where there is a high infection rate, all patients
Table 3 Suggested adjustments of trauma bay protocols during endemic COVID-19

\begin{tabular}{ll}
\hline Medical personnel & Appropriate Team Training to use PPE \\
& Consider every patient as infected \\
& Minimize direct contact and define "hands-on" and "hands-off" teams \\
& Define strategies in treatment of two and more patients simultaneously \\
& Cohort infected patients if possible \\
& Avoid transportation through hospital \\
& Use portable imaging \\
Diagnostics & Reduce or avoid repeated evaluations \\
& No face to face consultations \\
& Avoid second hit phenomenon in infected \\
& $\bullet$ Minimize interventions "damage control" \\
& $\bullet$ Adequate prevention of thrombosis \\
- Avoid secondary infection & - Lung-protective ventilation \\
\hline
\end{tabular}

PPE personal protective equipment 
need to be initially treated as infected until proven otherwise [12]. The number of medical personnel with direct contact to the patient needs to be decreased during such pandemics in order to increase patient and medical personnel safety [13]. Moreover, the trauma team needs appropriate training in the safe use of personal protective equipment (PPE) [14]. In order to avoid cross-contamination, the exact roles in trauma bay need to be adjusted for pandemics such as COVID-19 [15]. Physicians with direct contact to the patient (e.g., anesthesiologists and surgeons) may be defined as the "hands-on" team, whom perform the line placement, blood collection, and clinical examination or emergency interventions (Fig. 1). Depending on the injury pattern or severity, the initial team can be supplemented (hands-off team) with additional colleagues (e.g. neurosurgeons, vascular surgeons, thoracic surgeons etc.) (Fig. 1). The function and the role of a "trauma leader" stays unaffected according to each system and organization. Especially, if the treatment of more than one severely injured patients simultaneously requires a clear definition of teams and consideration of hygiene standards. Moreover, the above-mentioned strategies lead to a rationalized use of personal protective equipment, such as gloves, masks, and glasses.

\section{Diagnostics}

Severely injured patients are frequently subjected to different diagnostic modalities to identify life-threatening injuries and patterns. Chest and pelvis X-rays, sonography, whole-body computed tomography (WBCT), and angiography are commonly used diagnostic procedures in trauma bay. The evaluation of the trauma patient should not be delayed, and appropriate precautions should be taken. When possible, diagnostic procedures are performed at sites with less traffic of outpatients to avoid secondary exposure of patients and medical staff. When possible, COVID-19 patients should be placed in a separate location from non-COVID-19 patients; however, optimal injury care should be a priority [16]. Transportation of trauma patients through the hospital should be avoided. Therefore, whenever possible, the use of portable imaging, such as portable x-rays and sonography, is recommended. Every patient is assumed to be infected; therefore, adequate decontamination of imaging equipment and any surface is necessary. When possible, repeated examinations should be reduced or entirely avoided with initial adequate diagnostics. The number of patient transfers to imaging facilities can be reduced by performing all imaging directly upon admission. In order to increase capacity, non-urgent imaging can be delayed or postponed. Discussions of imaging referrals during face-to-face consultations have a potential risk of contamination, and it is recommended that this is done over the phone.

\section{General management}

Recent publications indicate that a relevant number of SARSCoV-2-infected patients may develop severe inflammatory complications with diffuse pulmonary inflammation and alveolar damage $[17,18]$. Some reports even document the presence of a cytokine storm in the infected patients leading to altered cellular and humoral immune responses [19-21], similar to post-traumatic acute lung injuries (ALIs) induced by both direct and non-direct pulmonary insults [22, 23]. Besides the assessment of the overall conditions of trauma patients (age, injury severity, pattern, physiological response), the presence of bilateral opacities in chest imaging may influence the decision-making in trauma bay, as well. The surgical

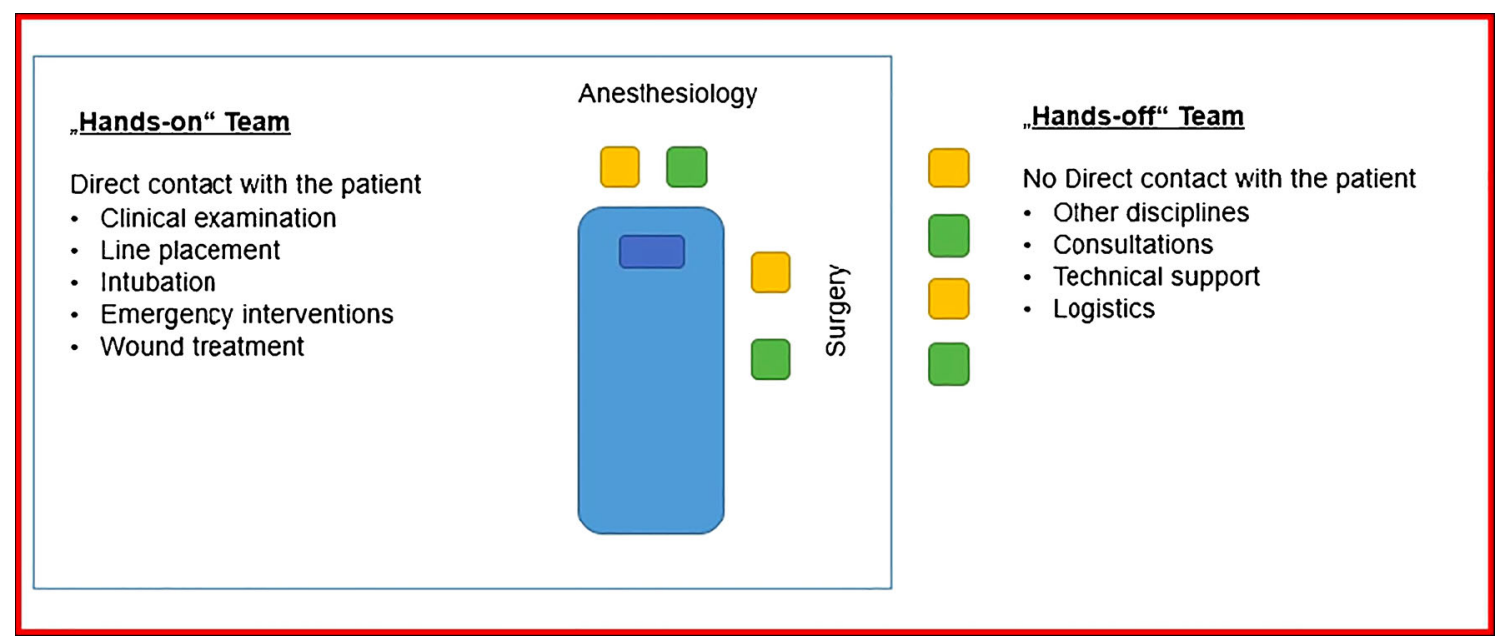

Fig. 1 The whole treatment team could be grouped in two teams: handson and hands-off teams. The hands-on team has a direct contact with the patient and performs clinical examination and emergency interventions.
The hands-off team avoids the direct contact with the patient and contaminated materials 
management should be based on minimizing the "secondary hit" phenomena to the immune response [24, 25]. In addition, lung-protective ventilation protocols may prevent barotrauma. Mechanical ventilation can exacerbate lung damage by causing a secondary induced lung injury when used improperly [26]. The prevention of secondary infections is also stressed in order to prevent additional infectious insults and inflammatory exaggerations. Moreover, recent publications indicate high rates of thromboembolic complications in COVID-19infected patients [27]. Therefore, adequate anticoagulation is recommended according to patient conditions.

\section{Conclusion}

The worldwide COVID-19 pandemic has had an immense impact on the day life and clinical practices. The number of patients admitted to the trauma bay has decreased; however, the number of severely injured trauma victims was unchanged. In order to maintain the quality standards, adjustment to the current trauma guidelines for pandemic situations is needed. Patient and personnel safety should be the number one priority in such situations. Moreover, the abovementioned strategies could also lead to improved and sustainable use of personal protective equipment.

Authors' contributions Sascha Halvachizadeh: writing and revision of the manuscript, collecting data, analysis, interpretation of data and statistics, searched literature.

Michel Teuben: critical revision of the manuscript, searched literature.

Till Berk: critical revision of the manuscript, interpretation of data.

Valentin Neuhaus: critical revision of the manuscript, interpretation of data.

Hans-Christoph Pape: interpretation of data and statistics, critical revision of the manuscript, supervision of the study.

Roman Pfeifer: analysis and interpretation of data, statistics, critical revision of the manuscript, data collection, development and supervision of the study, searched literature.

All the authors read and approved the final manuscript.

SH developed the study protocol, collected and analyzed the data, interpretation of the data, writing the original manuscript, and revisions; MT interpreted the data and revised the manuscript; TB interpreted the data, revised the manuscript; VN interpreted data, revised the manuscript, and developed the study idea; HCP interpreted the data, provided the infrastructure, revised the manuscript, developed the study idea, and supervised the project; RP developed the study idea, collected and interpreted the data, revised the manuscript, supervised the project, and wrote the original article.

Funding Information Open access funding provided by University of Zurich.

Data availability All data and material are available upon request from the corresponding author.

The datasets generated during and/or analyzed during the current study are available in the figshare repository https://doi.org/10.6084/m9. figshare. 12436814

\section{Compliance with ethical standards}

Ethical approval and consent to participate, consent for publication The local institutional review board and the cantonal ethics committee received a detailed study protocol and waved the requirement for ethical approval to conduct this study (Nr. 2020-00789).

The study protocol and the study were approved by the local institutional review board (IRB) (Nr. 2020-00789).

Consent to participate The requirement for additional consent to participate was waived by the local IRB (Nr. 2020-00789).

Conflict of interest The authors declare that they have no conflict of interest.

Open Access This article is licensed under a Creative Commons Attribution 4.0 International License, which permits use, sharing, adaptation, distribution and reproduction in any medium or format, as long as you give appropriate credit to the original author(s) and the source, provide a link to the Creative Commons licence, and indicate if changes were made. The images or other third party material in this article are included in the article's Creative Commons licence, unless indicated otherwise in a credit line to the material. If material is not included in the article's Creative Commons licence and your intended use is not permitted by statutory regulation or exceeds the permitted use, you will need to obtain permission directly from the copyright holder. To view a copy of this licence, visit http://creativecommons.org/licenses/by/4.0/.

\section{References}

1. WHO (2016) The top 10 causes of death. In: Organization WH (ed)

2. von Ruden C, Buhren V, Perl M (2017) Polytrauma management treatment of severely injured patients in ER and OR. Z Orthop Unfallchir 155:603-622. https://doi.org/10.1055/s-0042-124275

3. van Vugt R, Kool DR, Lubeek SFK, Dekker HM, Brink M, Deunk J, Edwards MJR (2013) An evidence based blunt trauma protocol. Emerg Med J 30:6. https://doi.org/10.1136/emermed-2011-200802

4. Rossaint R, Bouillon B, Cerny V, Coats TJ, Duranteau J, Fernandez-Mondejar E, Filipescu D, Hunt BJ, Komadina R, Nardi G, Neugebauer EAM, Ozier Y, Riddez L, Schultz A, Vincent JL, Spahn DR (2016) The European guideline on management of major bleeding and coagulopathy following trauma: fourth edition. Crit Care 20:55. https://doi.org/10.1186/s13054-016-1265$\mathrm{x}$

5. Bouillon B, Probst C, Maegele M, Wafaisade A, Helm P, Mutschler M, Brockamp T, Shafizadeh S, Paffrath T (2013) Emergency room management of multiple trauma : ATLS(R) and S3 guidelines. Chirurg 84:745-752. https://doi.org/10.1007/s00104-013-2476-1

6. Bouillon B, Marzi I (2018) The updated German "polytrauma guideline": an extensive literature evaluation and treatment recommendation for the care of the critically injured patient. Eur J Trauma Emerg Surg 44:S1-S1. https://doi.org/10.1007/s00068-018-0949-0

7. Mavragani A (2020) Tracking COVID-19 in Europe: an infodemiology study. JMIR Public Health Surveill. https://doi. org/10.2196/18941

8. The L (2020) COVID-19: protecting health-care workers. Lancet 395:922. https://doi.org/10.1016/S0140-6736(20)30644-9

9. von Elm E, Altman DG, Egger M, Pocock SJ, Gotzsche PC, Vandenbroucke JP, Initiative S (2014) The Strengthening the Reporting of Observational Studies in Epidemiology (STROBE) statement: guidelines for reporting observational studies. Int J Surg 12:1495-1499. https://doi.org/10.1016/j.ijsu.2014.07.013 
10. Copes WS, Champion HR, Sacco WJ, Lawnick MM, Keast SL, Bain LW (1988) The injury severity score revisited. J Trauma Acute Care Surg 28:69-77

11. Bouillon B, Pieper D, Flohe S, Eikermann M, Prengel P, Ruchholtz S, Sturmer K, Waydhas C, Trentzsch H, Lendemans S, HuberWagner S, Rixen D, Hildebrand F, Mosch C, Nienaber U, Sauerland S, Schenkel M, Walgenbach M, Becker M, Buhn S, He S, Jaschinski T, Mathes T, Bernhard M, Bottiger B, Burger T, Fischer M, Gutwald R, Hohenfellner M, Klar E, Rickels E, Schuttler J, Seekamp A, Swoboda L, Vogl T, Waldfahrer F, Wustner-Hofmann M, Bader W, Duran A, Gathof B, Geyer L, Haske D, Helm M, Hilbert-Carius P, Kanz KG, Kneser U, Lehnhardt M, Lier H, Lott C, Ludwig C, Marzi I, Mauer U, Schafer J, Schwab R, Siemers F, Strasser E, Wessel L, Wirth S, Wurmb T, Polytrauma Guideline Update G (2018) Level 3 guideline on the treatment of patients with severe/multiple injuries abstracts. Eur J Trauma Emerg Surg 44:S3-S271. https://doi.org/10. 1007/s00068-018-0922-y

12. Canarutto D, Priolo A, Russo G, Pitea M, Vigone MC, Barera G (2020) COVID-19 infection in a paucisymptomatic infant: raising the index of suspicion in epidemic settings. Pediatr Pulmonol. https://doi.org/10.1002/ppul.24754

13. Sanche S, Lin YT, Xu C, Romero-Severson E, Hengartner N, Ke R (2020) High contagiousness and rapid spread of severe acute respiratory syndrome coronavirus 2. Emerg Infect Dis 26. https://doi. org/10.3201/eid2607.200282

14. Forrester JD, Nassar AK, Maggio PM, Hawn MT (2020) Precautions for operating room team members during the COVID-19 pandemic. J Am Coll Surg. https://doi.org/10.1016/j. jamcollsurg.2020.03.030

15. Bin SY, Heo JY, Song MS, Lee J, Kim EH, Park SJ, Kwon HI, Kim SM, Kim YI, Si YJ, Lee IW, Baek YH, Choi WS, Min J, Jeong HW, Choi YK (2016) Environmental contamination and viral shedding in MERS patients during MERS-CoV outbreak in South Korea. Clin Infect Dis 62:755-760. https://doi.org/10.1093/cid/ civ1020

16. American College of Surgeons F Maintaining Trauma Center Access \& Care during the COVID-19 Pandemic: Guidance Document for Trauma Medical Directors. In: Trauma ACoSCo (ed)

17. Yang X, Yu Y, Xu J, Shu H, Xia J, Liu H, Wu Y, Zhang L, Yu Z, Fang M, Yu T, Wang Y, Pan S, Zou X, Yuan S, Shang Y (2020) Clinical course and outcomes of critically ill patients with SARSCoV-2 pneumonia in Wuhan, China: a single-centered, retrospective, observational study. Lancet Respir Med. https://doi.org/10. 1016/S2213-2600(20)30079-5

18. Xu Z, Shi L, Wang Y, Zhang J, Huang L, Zhang C, Liu S, Zhao P, Liu H, Zhu L, Tai Y, Bai C, Gao T, Song J, Xia P, Dong J, Zhao J, Wang FS (2020) Pathological findings of COVID-19 associated with acute respiratory distress syndrome. Lancet Respir Med 8: 420-422. https://doi.org/10.1016/S2213-2600(20)30076-X
19. Chien JY, Hsueh PR, Cheng WC, Yu CJ, Yang PC (2006) Temporal changes in cytokine/chemokine profiles and pulmonary involvement in severe acute respiratory syndrome. Respirology 11: 715-722. https://doi.org/10.1111/j.1440-1843.2006.00942.x

20. Zhang Y, Li J, Zhan Y, Wu L, Yu X, Zhang W, Ye L, Xu S, Sun R, Wang Y, Lou J (2004) Analysis of serum cytokines in patients with severe acute respiratory syndrome. Infect Immun 72:4410-4415. https://doi.org/10.1128/IAI.72.8.4410-4415.2004

21. Diao B, Wang C, Tan Y, Chen X, Liu Y, Ning L, Chen L, Li M, Liu Y, Wang GJM (2020) Reduction and functional exhaustion of $\mathrm{T}$ cells in patients with coronavirus disease 2019 (COVID-19)

22. Schimunek L, Serve R, Teuben MPJ, Stormann P, Auner B, Woschek M, Pfeifer R, Horst K, Simon TP, Kalbitz M, Sturm R, Pape HC, Hildebrand F, Marzi I, Relja B (2017) Early decreased TLR2 expression on monocytes is associated with their reduced phagocytic activity and impaired maturation in a porcine polytrauma model. PLoS One 12:16. https://doi.org/10.1371/ journal.pone. 0187404

23. Villar J, Zhang H, Slutsky AS (2019) Lung repair and regeneration in ARDS: role of PECAM1 and Wnt signaling. Chest 155:587594. https://doi.org/10.1016/j.chest.2018.10.022

24. Pape HC, Grimme K, van Griensven M, Sott AH, Giannoudis P, Morley J, Roise A, Ellingsen E, Hildebrand F, Wiese R, Krettek C (2003) Impact of intramedullary instrumentation versus damage control for femoral fractures on immunoinflammatory parameters: prospective randomized analysis by the EPOFF study group. J Trauma-Injury Infect Crit Care 55:7-13. https://doi.org/10.1097/ 01.Ta.0000075787.69695.4e

25. Giannoudis PV, van Griensven M, Hildebrand F, Krettek C, Pape HC (2008) Femoral nailing-related coagulopathy determined by first-hit magnitude - an animal study. Clin Orthop Relat Res 466: 473-480. https://doi.org/10.1007/s11999-007-0066-6

26. Nieman G, Satalin J, Andrews P, Wilcox K, Aiash H, Baker S, Kollisch-Singule M, Madden M, Gatto L, Habashi N (2018) Preemptive mechanical ventilation based on dynamic physiology in the alveolar microenvironment: novel considerations of timedependent properties of the respiratory system. J Trauma Acute Care Surg 85:1081-1091. https://doi.org/10.1097/TA. 0000000000002050

27. Klok FA, Kruip M, van der Meer NJM, Arbous MS, Gommers D, Kant KM, Kaptein FHJ, van Paassen J, Stals MAM, Huisman MV, Endeman H (2020) Incidence of thrombotic complications in critically ill ICU patients with COVID-19. Thromb Res. https://doi.org/ 10.1016/j.thromres.2020.04.013

Publisher's note Springer Nature remains neutral with regard to jurisdictional claims in published maps and institutional affiliations. 